\title{
Rapid Purification of Rabbit Reticulocyte Lipoxygenase for Electron Paramagnetic Spectroscopy Characterization of the Non-Heme Iron
}

\author{
Richard T. Carrolla, Jessica Muller ${ }^{a}$, Jennifer Grimm ${ }^{a}$, William R. Dunham ${ }^{b}$, Richard H. Sands ${ }^{b}$ and Max \\ O. Funk, Jr: $a_{s}$ *

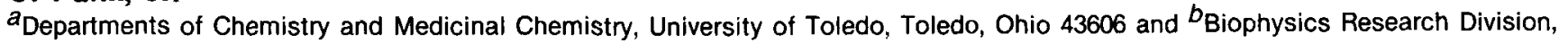 \\ University of Michigan, Ann Arbor, Michigan 48109
}

\begin{abstract}
An efficient three-step purification technique has been developed for the reticulocyte 15-lipoxygenase from rabbit. Ammonium sulfate fractionated reticulocyte lysate was purified by size exclusion chromatography and preparative scale isoelectric focusing. The entire procedure was complete in less than eight hours and was carried out in batches which typically yielded $10 \mathrm{mg}$ of purified enzyme. The identity and purity of the enzyme were evaluated by $N$-terminal sequencing, sodium dodecylsulfate polyacrylamide gel electrophoresis and specific activity determinations. The enzyme contained approximately one g-atom iron per mole of protein. The iron was present in an electron paramagnetic spectroscopy (EPR) silent, presumably high spin iron(II), form in the isolated enzyme. Treatment with one equivalent of 13 -hydroperoxy-9(Z),11(E). octadecadienoic acid resulted in the appearance of an EPR signal around $\mathrm{g} 6$.

Lipids 28, 241-244 (1993).
\end{abstract}

Lipoxygenase occupies a key position in the metabolism of polyunsaturated fatty acids in both plants and animals. The products of lipoxygenase catalysis and subsequent metabolites are potent mediators of an array of biochemical events. For example, there is a substantial body of evidence indicating that rabbit 15-lipoxygenase plays a central role in the maturation of the red blood cell (1). The enzyme apparently catalyzes the peroxidation of mitochondrial lipids resulting in the destruction of the organelle, the inaugural step in the conversion of the reticulocyte into the mature erythrocyte. Like all lipoxygenases, the reticulocyte enzyme contains one non-heme iron atom per molecule of the protein (2). While the reticulocyte enzyme was by a wide margin the first mammalian lipoxygenase to be isolated, the nonheme iron site has not been characterized. By contrast the non-heme iron site of soybean lipoxygenasel has been a central focus of studies of that enzyme. Features of the iron have been probed by ultraviolet (UV) visible, electron paramagnetic resonance (EPR), magnetic circular dichroism (MCD), Mössbauer, X-ray absorption, paramagnetic nuclear magnetic resonance (NMR) spectroscopy and magnetic susceptibility measurements $(3-5)$. These experiments demonstrated that the native soybean enzyme contained the metal in an EPR silent high-spin iron(II) form. Treatment of the native enzyme with the product of catalysis converted the metal to a high-spin iron(III) form with a characteristic EPR feature around g6. When the oxidized enzyme was

\footnotetext{
*To whom correspondence should be addressed at Department of Chemistry, University of Toledo, $2801 \mathrm{~W}$. Bancroft Street, Toledo, Ohio 43606.

Abbreviations: DTT, dithiothreitol; EDTA, ethylenediaminetetraacetic acid; EPR, electron paramagnetic spectroscopy; MCD, magnetic cir cular dichroism; NMR, nuclear magnetic resonance; PVDF, poly vinylidenedifluoride, SDS, sodium dodecylsulfate; UV, ultraviolet.
}

treated with linoleic acid (substrate) in the absence of oxygen, the EPR signal was lost, and a new iron(II) species was detected. Therefore it has been concluded that the unique non-heme iron site plays a fundamental redox role in the catalytic mechanism of the enzyme. The non-heme iron sites in porcine leukocyte 5- and 12-lipoxygenases have been recently investigated and also found to be EPR active (6). However, the leukocyte enzymes as isolated displayed an EPR signal around g5.2 which did not require and was not affeced by the product of catalysis. Also the signal was not abolished by treatment with a reducing agent, nordihydroguaiaretic acid. Two purification procedures for rabbit 15lipoxygenase have been published (2,7). While the procedures result in the isolation of homogeneous protein in good yields, there has been no apparent progress in the characterization of the non-heme iron site using spectroscopic probes. There is some indication that the purification scheme for the enzyme may need to be tailored to the specific application or investigation. For example, the purification procedure developed first was found not to provide protein samples suitable for crystallization. Initial experiments led us to believe that a critical element in the purification for the examination of the non-heme iron site might be speed. The previous procedures both involved multiple resolution steps with intervening concentration and dialysis manipulations spanning several days. Here we present a rapid purification procedure for the reticulocyte enzyme that provides samples of the enzyme suitable for undertaking the characterization of the non-heme iron site by EPR spectroscopy.

\section{MATERIALS AND METHODS}

Reticulocytes were obtained from New Zealand white rabbits injected (1.0-2.0 mL) daily for five days with a $1.2 \%$ aqueous solution of $N$-acetylphenylhydrazine adjusted to pH 6.8 using HEPES buffer $(1 M$, pH 7.0). The rabbits were bled daily from day 7 through day 12 . The amount of blood collected was between 30 and $50 \mathrm{~mL}$ per day and was adjusted to maintain a hematocrit level between 18 and $22 \%$ cells.

The supernatant from lysed cells (2) was brought to $55 \%$ saturation with ammonium sulfate, and the mixture was centrifuged. The pellet was redissolved in potassium phosphate buffer $(0.01 M, \mathrm{pH} 6.0)$ and was dialyzed against the same buffer at $5^{\circ} \mathrm{C}$. Precipitation was removed by centrifugation, and the supernatant was stored for future use in polypropylene tubes submerged in liquid nitrogen.

Samples (10-15 mL) of the thawed extracts were applied to a Sephacryl $S 200 \mathrm{HR}$ column $(2.5 \times 40 \mathrm{~cm})$ equilibrated with Tris buffer, $0.05 M, \mathrm{pH} 7.2$, containing $1 \mathrm{~m} M$ dithiothreitol (DTT) and $1 \mathrm{mM} \mathrm{MgCl}_{2}$. Elution was carried out with the same buffer at a flow rate of $2 \mathrm{~mL} \mathrm{~min}^{-1}$. During elution the buffer reservoir was purged continuously with nitrogen and was maintained at $5^{\circ} \mathrm{C}$. Fractions $(4$ $\mathrm{mL}$ ) were collected and stored under a nitrogen atmosphere on ice during subsequent analysis. Aliquots 
of the fractions were subjected to sodium dodecylsulfate (SDS) polyacrylamide determinations (8) and measurement of absorbance at 280 and $560 \mathrm{~nm}$.

Pooled fractions $(5,20 \mathrm{~mL})$ from the gel filtration experiment were combined with glycerol $(5 \mathrm{~mL}), 5 / 7 \mathrm{am}$ pholyte ( $3.0 \mathrm{~mL}$, Biorad, Richmond, CA), and ice-cold deionized water to give a final volume of $50 \mathrm{~mL}$. The sample was loaded into a preparative scale isoelectric focusing apparatus (Rotofor, Biorad), and a constant power (15 W) was applied for a period of four hours. The fractions were subjected to $\mathrm{pH}$, activity (2) and SDS polyacrylamide gel electrophoresis determinations as well as measurement of absorbance at 280 and $560 \mathrm{~nm}$. Fractions containing lipoxygenase were stored at $-20^{\circ} \mathrm{C}$ without loss of activity for up to two weeks.

Protein sequence determination was carried out on an Applied Biosystems model 477A liquid-pulsed peptide protein sequencer. Samples were analyzed for iron content using flame atomization atomic absorption spectrometry. EPR measurements were made at $9 \mathrm{GHz}$ using a Varian Century Line spectrometer (Palo Alto, CA) equipped with a liquid helium transfer line and a quartz dewar cavity insert. Spectra were recorded at $25 \mathrm{~K}$ using microwave power that avoided saturation. Running conditions were set at $5 \mathrm{~mW}$ power, $1 \mathrm{mT}$ modulation amplitude, $4 \mathrm{~min}$ scan time and $0.128 \mathrm{~s}$ time constant. The samples were prepared for analysis by dialysis against Tris buffer, containing $0.1 M \mathrm{NaCl}$ and $1 \mathrm{mM}$ ethylenediaminetetraacetic acid (EDTA). The oxidized enzyme was obtained by treatment with 13-hydroperoxy-9Z,11E-octadecadienoic acid (9) in a 1:1.1 ratio. Final concentration of the samples for EPR was carried out in a centrifugal concentrator (Amicon Centricon 30, Lexington, MA).

\section{RESULTS AND DISCUSSION}

The mammalian lipoxygenases are susceptible to inactivation with loss of iron in the presence of oxygen (absence of reducing agents) $(10,11)$. Therefore, a rapid purification procedure for rabbit 15-lipoxygenase which avoided exposure to oxygen was investigated. Attempted purification of the enzyme with a chromatofocusing procedure which has been successfully applied to the isolation of the soybean isoenzymes failed. A procedure based on size exclusion chromatography and preparative isoelectric focusing was developed.

The resolution of the proteins obtained from ammonium sulfate precipitated reticulocyte lysate by gel filtration chromatography is shown in Figure 1A. The applied sample was the result of a one-day collection of reticulocytes from two rabbits between day 7 and 14 of the protocol. The collected fractions were monitored for absorbance at 280 and $560 \mathrm{~nm}$ which allowed for detection of hemoglobin, a major protein component of the cells. The proteins were resolved between fractions 20 and 40 . When fractions in this region were tested for lipoxygenase activity, it was found that the hemoglobin interfered with the assay showing quasi-lipoxygenase activity. SDS polyacrylamide gel electrophoresis (Fig. 1B) showed that a protein of about $75 \mathrm{kDa}$ coeluted with hemoglobin. The only other candidate for the target enzyme in the elution profile was a protein of $70 \mathrm{kDa}$ that was present in fractions 24-28. These samples, however, displayed no lipoxygenase activity. The identity of the $75 \mathrm{kDa}$ protein as 15-lipoxy-

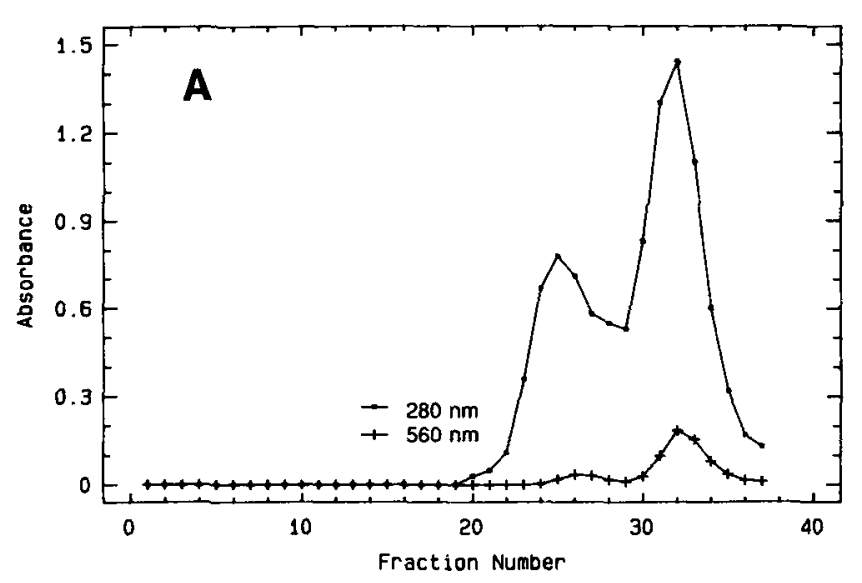

B

FRACTION NUMBER
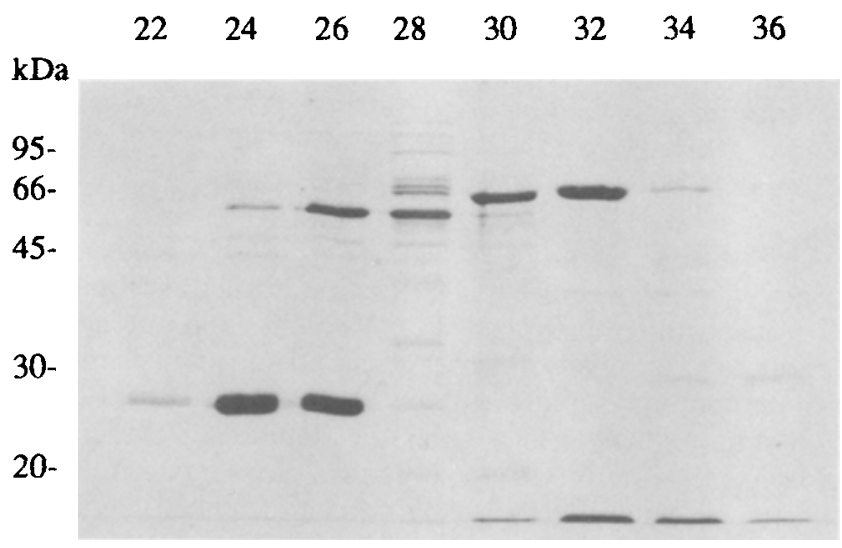

FIG. 1A. Elution profile for size exclusion chromatography of ammonium sulfate fractionated rabbit reticulocyte lysate. Sephacryl S200 HR, This $\mathrm{HCl}(0.05 M, \mathrm{pH} 7.2,1 \mathrm{~m} M$ dithiothreitol, $1 \mathrm{mM}$ $\mathrm{MgCl}_{2}$ ). B. Sodium dodecylsulfate polyacrylamide gel electrophoresis determinations of selected fractions from the size exclusion chromatography.

genase was confirmed by protein sequence analysis. The proteins in the gel were transferred electrophoretically to a polyvinylidenedifluoride (PVDF) membrane. After briefly staining and destaining the membrane containing the transferred proteins, the $75 \mathrm{kDa}$ band was excised and subjected to sequence determination using automated $\mathrm{Ed}$ man degradation chemistry. A clear amino acid sequence was obtained through 15 Edman cycles which matched exactly with the first 15 amino terminal residues for 15lipoxygenase deduced from the corresponding c-DNA (12). These observations showed that a simple size exclusion chromatographic experiment was sufficient to provide samples of the enzyme with the only primary contaminant being hemoglobin.

Preparative scale isoelectric focusing was used to resolve the lipoxygenase from the hemoglobin. The result of a typical isoelectric focusing experiment is presented in Figure 2A. The apparatus separated the sample into 20 fractions upon focusing which were monitored for absorbance at 280 and $560 \mathrm{~nm}$. The proteins were clearly resolved into two components one of which displayed 


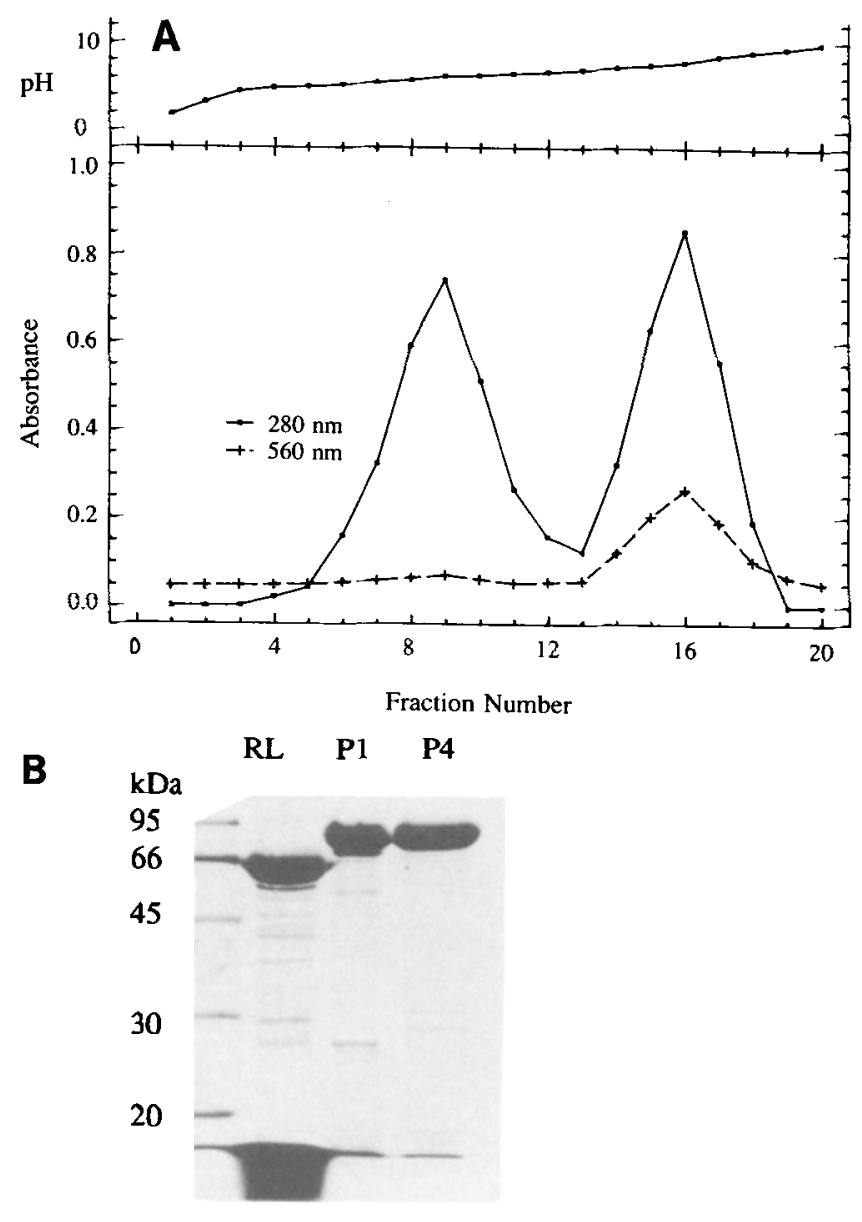

FIG. 2A. Preperative scale isoelectric focusing (pH 5-7) of fractions 30-34 from the size exclusion chromatography. B. Sodium dodecylsulfate polyacrylamide gel electrophoresis determination of fractions 8-10 (RL) from isoelectric focusing. Two isoenzymes of soybean lipoxygenase (P1, P4) were included for comparison.

significant absorbance at $560 \mathrm{~nm}$ due to the presence of hemoglobin. The other protein component was the $75 \mathrm{kDa}$ protein previously identified as 15-lipoxygenase. The procedure typically yielded approximately $10 \mathrm{mg}$ of the purified protein. The SDS polyacrylamide gel electrophoresis analysis of samples from fractions containing this protein (Fig. 2B; two isoenzymes of soybean lipoxygenase were run concurrently for comparison) showed the presence of only a single polypeptide and the ampholytes used in the separation procedure. This protein was found in a fraction with a measured $\mathrm{pH}$ of 5.5 , consistent with the previously reported $\mathrm{pI}$ for the 15 -lipoxygenase. The turnover number for lipoxygenase catalysis found for the fractions containing the $75 \mathrm{kDa}$ protein was $30 \mathrm{~s}^{-1}$, a value comparable to the reported $25 \mathrm{~s}^{-1}$ found previously for highly purified enzyme (2). The purified 15-lipoxygenase was also subjected to analytical isoelectric focus. ing determination in a polyacrylamide gel from $\mathrm{pH} 5$ to 7 (13). A single band staining for protein and activity at pH 7 was detected in the experiment (data not shown). These experiments demonstrated that useful quantities of 15-lipoxygenase were obtained in an isolation procedure taking less than eight hours.

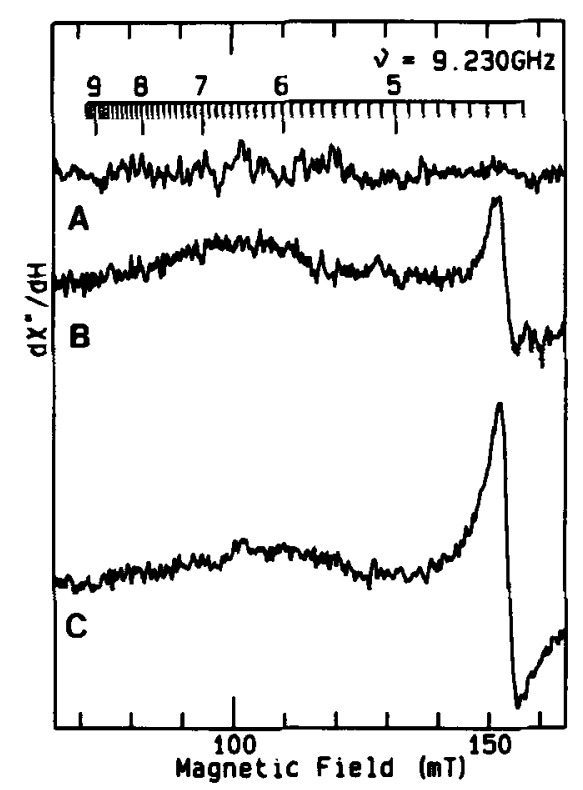

FIG. 3. Electron paramagnetic spectroscopy spectra for samples of purified reticulocyte lipoxygenase, Tris HCI (0.1 M, pH 7.4). (A) Spectrum of isolated enzyme, $200 \mu M$. (B) Isolated enzyme, $165 \mu M$, treated with 1.1X 13-hydroperoxy-9(Z),11(E)-octadecadienoic acid. (C) Oxidized enzyme, $200 \mu M$ purged with argon and treated with $1.2 \mathrm{X}$ linoleic acid.

For further experiments the enzyme solution was dialyzed in nitrogen purged Tris buffer $(0.1 \mathrm{M}, \mathrm{pH} 7.4,1 \mathrm{mM}$ EDTA) at $5^{\circ} \mathrm{C}$ and concentrated in a centrifugal concentrator. These procedures were not accompanied by precipitation of protein. Solutions were obtained with concentrations of the enzyme around $0.20 \mathrm{mM}$ displaying undiminished turnover number $\left(30 \mathrm{~s}^{-1}\right)$. Samples of the purified and dialyzed enzyme contained $0.9 \mathrm{~g}$-atom of iron per mole of enzyme using flame atomization atomic absorption spectrometry. The UV-visible spectrum of the purified enzyme contained only the absorbance maximum around $280 \mathrm{~nm}$ expected for the contribution of aromatic amino acids. Treatment of the enzyme solution with one equivalent of 13-hydroperoxy-9(Z),11(E)-octadecadienoic acid caused an increase in absorbance in the region 300$350 \mathrm{~nm}$, but no new maximum was obtained. The UVvisible spectroscopy was, therefore, similar to observations on lipoxygenase-1 from soybeans (4). The enzyme as isolated had spectroscopic features consistent with a nonheme iron atom present in a high spin iron(II) form that was oxidized upon treatment with the product of catalysis to give a high spin iron(III) species.

The characterization of the non-heme iron site in the reticulocyte 15-lipoxygenase by EPR spectroscopy is presented in Figure 3. No signals were detected in the solution of the enzyme obtained from the isolation procedure (Trace A). This was consistent with the iron being present in the protein as high spin iron(II) as has been found and confirmed in other spectroscopic measurements for the soybean enzyme. Treatment of the enzyme with 13-hydroperoxy-9(Z),11(E)-octadecadienoic acid resulted in the appearance of two new signals in the EPR spectrum (Trace B), a sharp signal at 94.3 and a broad featureless signal around $g 6$. The integrated intensity of the g6 signal accounted for $15-20 \%$ of the iron known to be in the sample. 
When samples of the enzyme treated with product were made anaerobic by purging with argon and were subsequently treated with linoleic acid, the spectrum changed (Trace C). There was a diminution in the g6 signal and an increase in the signal at g4.3. The EPR observations were similar in nature to those for the soybean enzyme, but different in degree. The soybean enzyme was converted stoichiometrically into a high spin iron(III) species with the g6 EPR signal by oxidation with product, and the signal was abolished by treatment with substrate in the absence of oxygen (14). We conclude from the similarities in the UV-visible and EPR spectroscopy for the two enzymes that the non-heme iron sites in reticulocyte and soybean 15-lipoxygenase are similar. We speculate that aggregation of the reticulocyte enzyme in the concentrated solutions used in the spectroscopic studies resulted in diminished accessibility of reagents to the active/iron site resulting in incomplete reactions. The development of an efficient precedure for obtaining significant quantities of highly purified rabbit 15-lipoxygenase should facilitate the characterization of the physical and catalytic properties of this important enzyme.

\section{ACKNOWLEDGEMENT}

This research was supported by a grant from the National Science Foundation (DMB 87-13537).

\section{REFERENCES}

1. Rapoport, S.M. (1986) The Reticulocyte, CRC Press, Boca Raton.

2. Rapoport, S.M., Schewe, T., Wiesner, R., Halangk, W., Ludwig, P., Janicke-Höne, M., Tannert, C., Hiebsch, C., and Klatt, D. (1979) Eur. J. Biochem. 96: 545-559.
3. Funk, M.O., Carroll, R.T., Thompson, J.F., Sands, R.H., and Dunham, W.R. (1990) J. Am. Chem. Soc 112, 5375-5376.

4. Feiters, M.C., Boelens, H., Veldink, G.A., Vliegenthart, J.F.G., Navaratnam, S., Allen, J.C., Nolting, H.F., and Hermes, C. (1990) Recl. Trav. Chim. Pays-Bas 109, 133-146.

5. Zhang, Y., Gebhard, M.S., and Solomon, E.I. (1991) J. Am. Chem. Soc. 113, 5162-5175.

6. Kroneck, P.M.H., Cucurou, C., Ullrich, V., Ueda, N., Suzuki, H., Yoshimoto, T., Matsuda, S., and Yamamota S. (1991) FEBS Lett. 287, 105-107.

7. Sloane, D.L., Browner, M.F., Dauter, Z., Wilson, K., Fletterick, R.J., and Sigal, E. (1990) Biochem. Biophys. Res. Commun. 173, 507-513.

8. Laemmli, U.K. (1970) Nature 227, 680-685.

9. Funk, M.O., Isaac, R., and Porter, N.A. (1976) Lipids 11, 113117.

10. Percival, D.M. (1991) J. Biol. Chem. 266, 10058-10061.

11. Höne, W.E., Kojima, N., Thiele, B., and Rapoport, S.M. (1991) Biomed. Biochim. Acta 50, 125-138.

12. O'Prey, J., Chester, J., Thiele, B.J., Janetzki, S., Prehn, S., Fleming, H., and Harrison, P.R. (1989) Gene 84, 493-499.

13. Funk, M.O., Whitney, M.A., Hausknecht, E.C., and O'Brien, E.M. (1985) Anal. Biochem. 146, 246-251.

14. de Groot, J.J.M.C., Veldink, G.A., Vliegenthart, J.G.G., Boldingh, J., Wever, R., and van Gelder, B.F. (1975) Biochim. Biophys. Acta $377,71-79$.

[Received July 14, 1992, and in revised form October 14, 1992; Revision accepted November 30, 1992] 